\title{
Interdisciplinary Diagnosis and Management of Cerebrospinal Fluid Leakage Due to a Meningeal Diverticulum: a Case Report
}

\author{
Christoph Gregor Trumm ${ }^{1}$ (D) $\cdot$ Robert Forbrig $^{1}$
}

Accepted: 10 December 2020 / Published online: 19 December 2020

(C) The Author(s) 2020

\begin{abstract}
Cerebrospinal fluid leakage through meningeal diverticula represents a diagnostic and therapeutic challenge comparatively rarely encountered in the interdisciplinary management of spontaneous intracranial hypotension (SIH). Several false-positive CSF leakage signs may be observed during the imaging work-up of SIH. A 27-year-old female with orthostatic headache showing marked spinal epidural CSF collections and MRI signs of intracranial hypotension underwent a blind and CT-guided epidural blood patch (EBP) of a pathological T9/10 meningeal diverticulum (MD), detected by dynamic CT myelography (dCTM). After initial good imaging and symptomatic improvement, recurrent symptoms and a large left-sided subdural hematoma required neurosurgical MD ligation, with persisting clinical success. The following aspects of this brief report are remarkable: added value of dCTM to synchronously detect true CSF leakage and false-positive CSF leakage signs, near-complete resolution of spinal epidural CSF collections after CT fluoroscopy-guided EBP, interdisciplinary diagnosis, and definite management of CSF leakage through an anomalous MD.
\end{abstract}

Keywords Case report $\cdot$ Computed tomography guidance $\cdot$ Epidural blood patch $\cdot$ Intracranial hypotension $\cdot$ Orthostatic headache

$\begin{array}{ll}\text { Abbreviations } \\ \text { C } & \text { Cervical } \\ \text { CISS } & \text { Constructive interference in steady state } \\ \text { cMRI } & \text { Cranial magnetic resonance imaging } \\ \text { CSF } & \text { Cerebrospinal fluid } \\ \text { CTF } & \text { Computed tomography fluoroscopy } \\ \text { dCTM } & \text { Dynamic computed tomography myelography } \\ \text { DSM } & \text { Digital subtraction myelography } \\ \text { EBP } & \text { Epidural blood patch }\end{array}$

Key Points

- Added value of dCTM to synchronously detect true CSF leakage and false-positive CSF leakage signs.

- Near-complete resolution of spinal epidural CSF collections after CT fluoroscopy-guided EBP.

- Interdisciplinary diagnosis and definite management of CSF leakage through an anomalous MD.

This article is part of the Topical Collection on Imaging

Robert Forbrig

robert.forbrig@med.uni-muenchen.de

Christoph Gregor Trumm

christoph.trumm@med.uni-muenchen.de

1 Institute of Neuroradiology, University Hospital, LMU Munich, Marchioninistrasse 15, 81377 Munich, Germany

$\begin{array}{ll}\text { G } & \text { Gauge } \\ \text { ILF } & \text { Interlaminar fenestration } \\ \text { L } & \text { Lumbar } \\ \text { MD } & \text { Meningeal diverticulum } \\ \mathrm{mL} & \text { Milliliter } \\ \mathrm{S} & \text { Sacral } \\ \mathrm{SDH} & \text { Subdural hematoma } \\ \mathrm{SIH} & \text { Spontaneous intracranial hypotension } \\ \mathrm{sMRI} & \text { Spinal magnetic resonance imaging } \\ \mathrm{T} & \text { Thoracic } \\ \mathrm{T} 1 \mathrm{w} & \text { T1-weighted imaging } \\ \mathrm{T} 2 \mathrm{w} & \text { T2-weighted imaging }\end{array}$

\section{Introduction}

Spontaneous intracranial hypotension (SIH) due to cerebrospinal fluid (CSF) leakage may become manifest in a variety of symptoms such as orthostatic headache, nausea, neck pain, and cranial nerve palsy with visual impairment up to sensorineural hearing loss [1]. By definition SIH patients have no clear history of trauma although minor trauma can often be found when exploring patient anamnesis in detail. Many SIH cases are successfully managed using conservative treatment 
without or with one or several epidural blood patches (EBP). If the actual CSF leakage site is detected during imaging work-up of SIH, CT guidance may be advantageous for targeted EBP application. Atypical meningeal diverticulum (MD) represents a potential anatomic site of CSF leakage and consecutive SIH. So far, only few authors described the successful treatment of those symptomatic MDs with EBP only or surgical ligature [2-4], while the particular utilization of CT fluoroscopy (CTF) guidance for targeted EBP was only reported in a large unselected series of CSF leakage cases [5].

\section{Case Presentation}

We report the case of a 27-year-old woman with chronic migraine who presented with a 1-month history of orthostatic headache. Further symptoms included tinnitus, nausea, and sensitivity to light. Apart from a recent manual treatment and yoga 2 weeks prior to admission, the patient denied any trauma. Initial cranial magnetic resonance imaging (cMRI) showed bilateral subdural hygromas, venous distension, and an increased pachymeningeal enhancement (Fig. 1a), consistent with SIH. Spinal MRI (sMRI) revealed several findings: (a) a marked fluid collection in the extradural space and suboccipital soft tissue at the $\mathrm{C} 1 / 2$ level, (b) a narrow ventral epidural CSF collection at C7/T1 (Fig. 1b) and L3 to S1, and a more accentuated dorsal epidural cerebrospinal fluid (CSF) collection extending from T1 to L1 (Fig. 1b), and (c) a conspicuous CSF collection at the left T9/10 neuroforamen (Fig. 1c). Because conservative therapy with caffeine, hydrocortisone, and analgesics did not yield a satisfactory result, a lumbar epidural blood patch (EBP) with 20 milliliters (mL) of autologous blood was carried out but did also not further alleviate clinical symptoms. Thus, on day 8 , dynamic computed tomography myelography (dCTM) was performed using $15 \mathrm{~mL}$ of undiluted Imeron $300 \mathrm{M}$ (Iomeprol, Bracco Imaging, Konstanz, Germany) to locate the origin of the assumed CSF leak which clearly identified CSF extravasation at T9/10 (Fig. 1d, e). Using a dedicated CT scanner (BrightSpeed S, GE Healthcare, Chicago, IL, USA), three dynamic (i.e., repetitive) CT scans after $10 \mathrm{~min}$ (mid T7 to $\mathrm{S} 1$; scan range $33.3 \mathrm{~cm}$ ), $15 \mathrm{~min}$ (posterior cranial fossa to $\mathrm{T} 10 ; 33.5 \mathrm{~cm}$ ), and $25 \mathrm{~min}$ (posterior cranial fossa to $\mathrm{T} 1 / 2$; $17.8 \mathrm{~cm}$ ) depicted a continuous ascent of contrast medium within the thoracic and cervical epidural space, with consecutive bilateral extraforaminal CSF leakage at the cervicothoracic junction (Fig. 1f) and extensive CSF leakage into the suboccipital soft tissue at $\mathrm{C} 1 / 2$ (Fig. 1g). A targeted autologous EBP at the segment T9/10 was performed. Using lumbar intrathecal contrast administration, conventional CTM was obtained immediately prior to the intervention. Under CT fluoroscopy guidance, a 20-G Chiba needle was advanced into the dorsal epidural space and $18 \mathrm{~mL}$ of autologous blood was injected (Fig. 2a). Postinterventional sMRI on day 4 depicted a marked reduction of both the suboccipital and thoracic epidural CSF collection (Fig. 2b, c). Correspondingly, at discharge on day 16 , the patient reported improvement of her orthostatic headache and remaining symptoms. However, 3 weeks later, she was readmitted with recurrent orthostatic headache. cMRI showed bilateral subdural hematoma (SDH) with a marked left-sided mass effect (Fig. 2d) and resulting parahippocampal herniation (not shown). sMRI still displayed reduction of the dorsal epidural CSF collection when compared to the pre-EBP sMRI (not shown). On day 3 after second admission, the patient underwent burr hole trepanation for drainage of the left-sided SDH (not shown), as well as leftsided interlaminar fenestration (ILF) at T9/10 (Fig. 2e, f) which confirmed a large CSF leak through the MD. Consequently, the MD was ligated at the transition zone of the nerve root and sealed with DuraGen ${ }^{\circledR}$ and TachoSil ${ }^{\circledR}$. MRI obtained 11 days after surgery showed a complete resolution of the SDH (not shown) as well as the spinal epidural CSF collections (Fig. 2g, h). Apart from discrete nausea, the patient was discharged free of symptoms.

\section{Conclusions}

We present a case of SIH with CSF leakage through a MD at T9/10, successfully located by $\mathrm{dCTM}$, temporarily treated with clinical success using a CT fluoroscopy-guided autologous EBP, and definitely by means of surgical repair after recurrence of symptoms and pronounced left-sided SDH. In the majority of SIH patients, the underlying CSF leakage occurs spontaneously and is commonly detected in sMRI [6]. The disease is typically self-limiting and conservative therapy with or without EBPs - applied either into the epidural space at the lower thoracic or lumbar level-frequently results in clinical improvement [1]. According to Leibold and colleagues, conservative therapy alone yields a $90 \%$ and $70 \%$ improvement of symptoms after four and $72 \mathrm{~h}$, respectively [7]. If these therapeutic measures fail, localization of the exact CSF leakage site is necessary by applying more sophisticated or invasive treatment such as targeted image-guided EBP or surgical repair. Though conventional CTM represents a commonly used modality for CSF leakage detection, some patients exhibit substantial CSF leaks that can be missed by CTM. Digital subtraction myelography (DSM) and dCTM have been proposed for localization of those rapid CSF leaks [8]. Although DSM is characterized by an excellent temporal resolution, this method should be performed under general anesthesia in order to obtain optimal results [9]. Thus, dCTM is the preferred diagnostic tool in most cases due to a higher availability and compatibility during clinical routine - in spite of the radiation exposure particularly to be considered in young patients when 
Fig. 1 a cMRI on patient admission showing signs of intracranial hypotension (axial gadolinium-enhanced T1w). Marked bilateral pachymeningeal enhancement (arrows). b, c sMRI on admission (3D CISS). Thin ventral cervicothoracic (arrow) and lumbar epidural CSF collection and marked dorsal

thoracolumbar epidural CSF collection (framed arrow). CSF collection at left T9/10 neuroforamen (asterisk). d, e Dynamic CT myelography. CT scan (lower thoracic and lumbar spine) immediately after lumbar intrathecal contrast administration $(15 \mathrm{~mL}$ Imeron ${ }^{\circledR} 300 \mathrm{M}$ ) and

Trendelenburg position: early contrast extravasation into MD at left T9/10 neuroforamen (asterisk) and dorsal epidural space (arrows). f CT scan after 25 min showing bilateral CSF extravasation at $\mathrm{C} 6 / 7$ and $\mathrm{C} 7 / \mathrm{T} 1$ ("false localizing cervicothoracic CSF extravasation sign") (arrows). Note the contrast-filled ventral and dorsal epidural space (framed arrow). g CT scan (cervical spine) 25 min after contrast administration: pronounced secondary CSF extravasation from epidural space into suboccipital soft tissue ("false localizing C1/2 sign") (arrows). Note the contrastfilled ventral and dorsal epidural space (framed arrows)
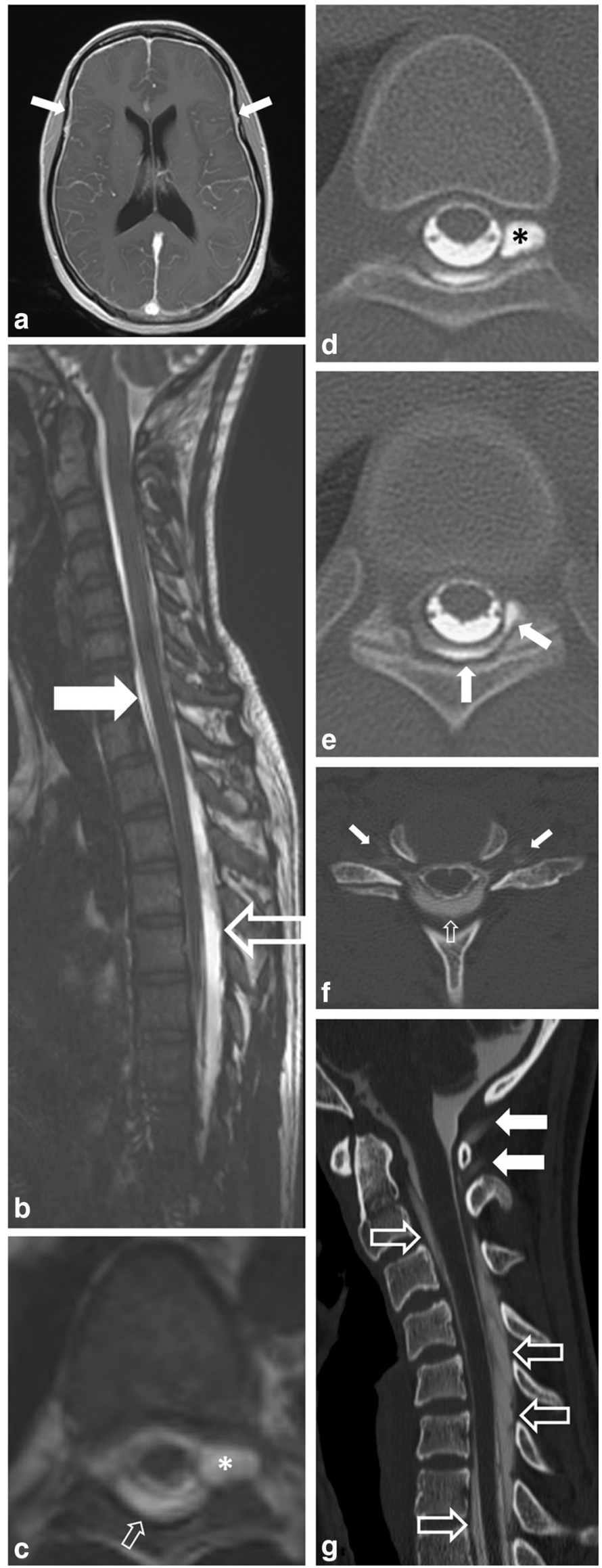

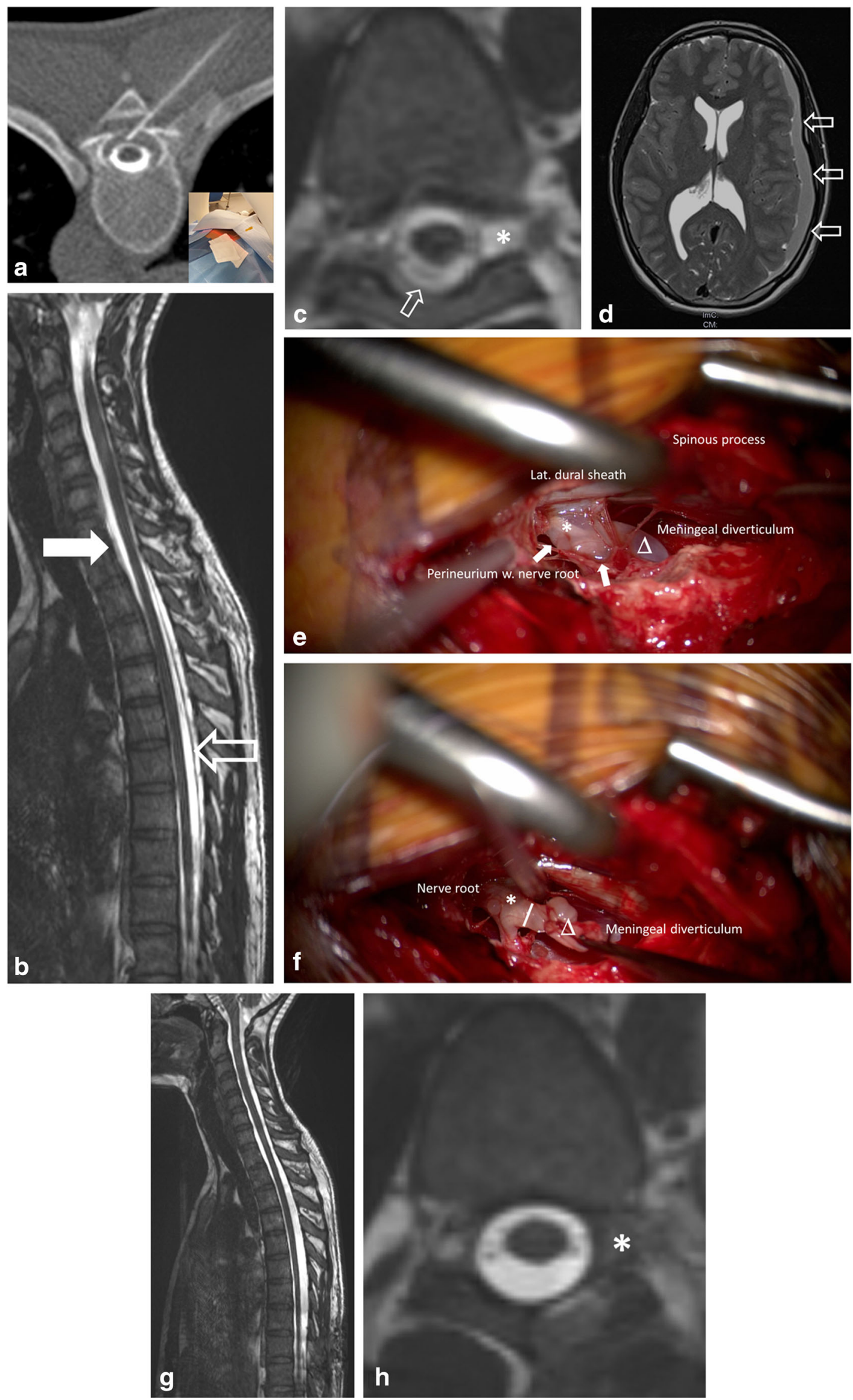
Fig. 2 a CT fluoroscopy-guided autologous EBP. Insertion of a 20-G Chiba needle into the dorsal epidural space at T9/10. b, c sMRI 4 days after EBP (3D CISS). Reduction of ventral cervicothoracic and lumbar CSF collection (arrow), and dorsal thoracolumbar epidural CSF collection (framed arrow). d cMRI 28 days after CT-guided EBP (axial T2w). Marked left-sided SDH (framed arrows). e Intraoperative image: thinnedout nerve root (asterisk) at T9/10 visible through the perineurium (arrows) with irregular adhesions. Note the neighboring $\operatorname{MD}(\Delta)$. $\mathbf{f}$ The $\operatorname{MD}(\Delta)$ is ligated at the transition zone (line) from the thinned-out and cystic nerve root (asterisk). $\mathbf{g}$, h sMRI (3D CISS). Complete resolution of cervicothoracic and thoracolumbar epidural CSF collections. Note the absence of the $\mathrm{MD}$ at $\mathrm{T} 9 / 10$ (asterisk)

determining the coverage of the CT scan. In our case, dCTM (three repeated scans as described above) yielded a total dose length product of $1438 \mathrm{mGy} \mathrm{cm}$ which is in the range of previously reported data [10].

In our patient, CSF leakage at the level of the T9/10 MD had already been suspected after planning MRI with highresolution thin-sliced T2w $3 \mathrm{D}$ images and was verified during the first dCTM scan covering the thoracolumbar spine after intrathecal contrast administration and Trendelenburg positioning. In two subsequent dCTM scans of the cervicothoracic spine (after 15 and $25 \mathrm{~min}$ ), the patient exhibited typical falsepositive CSF leakage signs: into the retrospinal soft tissue at $\mathrm{C} 1 / 2$ (already evident as fluid collection in initial sMRI) [11] and at the cervico-thoracic junction [12]. Yousry and colleagues were the first to describe the aforementioned retrospinal fluid collections at $\mathrm{C} 1 / 2$ in $\mathrm{SIH}$ patients as well as following dural puncture [13]. The authors hypothesized that these fluid collections are an ex- or transudate from dilated epidural veins. In contrast to few case reports describing true posttraumatic dural CSF leaks at C1/2 (e.g., [2]), Schievink et al. demonstrated that these fluid collections normally occur due to a CSF leak at a lower spinal level, distribute cranially within the spinal canal, and leave the epidural space into the soft tissue at $\mathrm{C} 1 / 2$ [11]. This mechanism was clearly depicted in our case using repeated CT scans of the cervical spine 15 and $25 \mathrm{~min}$ after intrathecal contrast administration. Similar to the other false-positive CSF leakage sign, which is typically seen at the cervico-thoracic junction [12], a retrospinal CSF collection at $\mathrm{C} 1 / 2$ should not be mistaken for the actual site of CSF extravasation. Nevertheless, true CSF leakage was finally confirmed at the anomalous T9/10 nerve root in conjunction with a MD. Comparable cases of symptomatic CSF leaks through meningeal diverticula have been reported [2-4]. Successful management includes EBP alone $[2,3]$ and surgical ligature, with $[3,4]$ or without [3] preceding EBP. Despite initial clinical and imaging response, in our patient, the dural defect at the left $\mathrm{T} 9 / 10$ nerve root assumedly got occluded only temporarily after targeted EBP, with a rebound effect of the intracranial hygromas converting to SDH that required burr hole trepanation, as well as definitive surgical ligation of the MD.
Acknowledgments We thank Dr. Raimund Trabold, Department of Neurosurgery, University Hospital, LMU Munich, Germany, for contributing intraoperative photographs.

Authors' Contributions C.G.T. and R.F. were responsible for data collection and interpretation. C.G.T. drafted the manuscript and prepared the figures. C.G.T. and R.F. revised the manuscript.

Funding Open Access funding enabled and organized by Projekt DEAL.

Data Availability Data and material are available upon reasonable request.

\section{Compliance with Ethical Standards}

Conflict of Interest The authors declare that they have no conflict of interest.

Ethics Approval Ethics approval was obtained prior to writing of this case report (Project ID 20-342 KB)

Consent to Participate Written informed consent and consent to participate was provided by the patient.

Consent for Publication Written consent for publication was provided by the patient.

Code Availability Not applicable

Open Access This article is licensed under a Creative Commons Attribution 4.0 International License, which permits use, sharing, adaptation, distribution and reproduction in any medium or format, as long as you give appropriate credit to the original author(s) and the source, provide a link to the Creative Commons licence, and indicate if changes were made. The images or other third party material in this article are included in the article's Creative Commons licence, unless indicated otherwise in a credit line to the material. If material is not included in the article's Creative Commons licence and your intended use is not permitted by statutory regulation or exceeds the permitted use, you will need to obtain permission directly from the copyright holder. To view a copy of this licence, visit http://creativecommons.org/licenses/by/4.0/.

\section{References}

1. Urbach H. Intracranial hypotension. Nervenarzt. 2014;85(8):102131; quiz 32. https://doi.org/10.1007/s00115-013-3849-x.

2. Armstrong N, Williamson C, Williamson N, Fortes M, Tjauw I, Vij $\mathrm{V}$, et al. Dural diverticulum with a symptomatic cerebrospinal fluid leak. Radiol Case Rep. 2016;11(1):16-20. https://doi.org/10.1016/ j.radcr.2015.10.001.

3. Enomoto N, Mure H, Okazaki T, Azumi M, Okita S, Nagahiro S, et al. Posttraumatic cerebrospinal fluid leak associated with an upper cervical meningeal diverticulum. World Neurosurg. 2018;116: 50-5. https://doi.org/10.1016/j.wneu.2018.05.061.

4. Lopez AJ, Campbell RK, Arnaout O, Curran YM, Shaibani A, Dahdaleh NS. Spontaneous cerebrospinal fluid leak from an anomalous thoracic nerve root: case report. J Neurosurg Spine. 2016;25(6):685-8. https://doi.org/10.3171/2016.4.SPINE151465.

5. Amrhein TJ, Befera NT, Gray L, Kranz PG. CT fluoroscopyguided blood patching of ventral CSF leaks by direct needle 
placement in the ventral epidural space using a transforaminal approach. AJNR Am J Neuroradiol. 2016;37(10):1951-6. https://doi. org/10.3174/ajnr.A4842.

6. Mokri B. Spontaneous cerebrospinal fluid leaks: from intracranial hypotension to cerebrospinal fluid hypovolemia-evolution of a concept. Mayo Clin Proc. 1999;74(11):1113-23. https://doi.org/ 10.4065/74.11.1113.

7. Leibold RA, Yealy DM, Coppola M, Cantees KK. Post-duralpuncture headache: characteristics, management, and prevention. Ann Emerg Med. 1993;22(12):1863-70.

8. Luetmer PH, Schwartz KM, Eckel LJ, Hunt CH, Carter RE, Diehn FE. When should I do dynamic CT myelography? Predicting fast spinal CSF leaks in patients with spontaneous intracranial hypotension. AJNR Am J Neuroradiol. 2012;33(4):690-4. https://doi.org/ 10.3174/ajnr.A2849.

9. Hoxworth JM, Patel AC, Bosch EP, Nelson KD. Localization of a rapid CSF leak with digital subtraction myelography. AJNR Am J Neuroradiol. 2009;30(3):516-9. https://doi.org/10.3174/ajnr. A1294.

10. Dobrocky T, Mosimann PJ, Zibold F, Mordasini P, Raabe A, Ulrich $\mathrm{CT}$, et al. Cryptogenic cerebrospinal fluid leaks in spontaneous intracranial hypotension: role of dynamic CT myelography. Radiology. 2018;289(3):766-72. https://doi.org/10.1148/radiol. 2018180732.

11. Schievink WI, Maya MM, Tourje J. False localizing sign of C1-2 cerebrospinal fluid leak in spontaneous intracranial hypotension. $\mathrm{J}$ Neurosurg. 2004;100(4):639-44. https://doi.org/10.3171/jns.2004. 100.4.0639.

12. Schievink WI, Maya MM, Chu RM, Moser FG. False localizing sign of cervico-thoracic CSF leak in spontaneous intracranial hypotension. Neurology. 2015;84(24):2445-8. https://doi.org/10.1212/ WNL.0000000000001697.

13. Yousry I, Forderreuther S, Moriggl B, Holtmannspotter M, Naidich TP, Straube A, et al. Cervical MR imaging in postural headache: MR signs and pathophysiological implications. AJNR Am J Neuroradiol. 2001;22(7):1239-50.

Publisher's Note Springer Nature remains neutral with regard to jurisdictional claims in published maps and institutional affiliations. 